\title{
Degree-of-Polarization and Eye-Closure Penalty Associated With Optical Signals With Orthogonal Polarizations
}

\author{
W. Shieh, R. Hui, and X. Yi
}

\begin{abstract}
We perform a systematic measurement of the degree-of-polarization (DOP) and eye-closure penalty for optical signals with orthogonal polarizations. We find that the symmetry of DOP is maintained for the orthogonal polarizations under both first and higher order polarization-mode dispersion (PMD), whereas the symmetry of eye-closure penalty is broken under second-order PMD. An orthogonal polarization pair can have large disparity of eye-closure penalty despite an identical DOP. We also demonstrate a novel approach to estimate the maximum eye-closure penalty asymmetry with three orthogonal polarizations on the Poincaré sphere.
\end{abstract}

Index Terms-Optical communication, optical dispersion, optical fiber polarization, optical fibers, polarization-mode dispersion (PMD).

\section{INTRODUCTION}

A S OPTICAL transmission systems advance toward ultralong reach and ultrahigh channel speed, polarization-mode dispersion (PMD) has become an active research and practical topic after Poole and Wagner's seminal work [1]. The PMD distorts optical signal waveform and places a fundamental limit on the channel speed [2]. Additionally, under the influence of PMD, the optical signal will experience polarization dispersion and its degree-of-polarization (DOP) will degrade. Such DOP degradation has been proposed as an indicator to monitor fiber PMD [3]. On the other hand, when an optical signal traverses a PMD impaired medium, it has been rigorously proved that despite the effect of depolarization, the Stokes vectors of two input orthogonal polarizations will be an inversion of each other at the output, i.e., the directions of the Stokes vector are opposite while their the magnitudes are equal [4]. In other words, the DOP (the magnitude of the normalized Stokes vector) is symmetric for the two orthogonal polarizations for any order of PMD. However, there is no systematic experimental verification for this theory so far. Furthermore, it has been shown that the second-order PMD-induced system impairment can be considered as the interference between the second-order PMD vector and fiber chromatic dispersion or phase chirp [5]. The same principle was successfully applied to the higher order PMD compensation [6]. The existence of

Manuscript received November 14, 2005; revised January 11, 2006. This work was supported by the Australian Research Council (ARC).

W. Shieh and X. Yi are with the ARC Special Research Centre for Ultra-Broadband Information Networks and National ICT Australia, Department of Electrical and Electronic Engineering, The University of Melbourne, Melbourne, VIC 3010, Australia (e-mail: w.shieh@ee.unimelb.edu.au).

R. Hui is with the Electrical Engineering and Computer Science Department, The University of Kansas, Lawrence, KS 66045-7621, USA.

Digital Object Identifier 10.1109/LPT.2006.873351

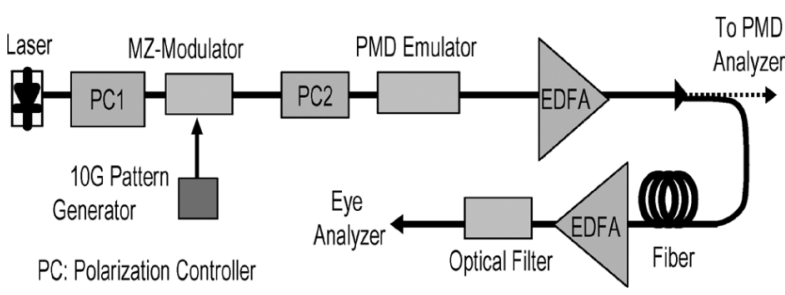

Fig. 1. Experimental setup for the symmetry study of orthogonal polarizations.

second-order PMD will consequently break the symmetry for the system penalty, i.e., the system penalties are no longer identical for two orthogonal polarizations, because the penalty is dependent on whether the input polarization is in- or out-ofphase with the second-order PMD vector [5]. In this letter, we perform systematic experimental study on the symmetry for orthogonal polarizations in terms of DOP and system penalty. We find that the symmetry of DOP is maintained for the orthogonal polarizations under both first-order and higher order PMD, whereas the symmetry of eye-closure penalty is broken under second-order PMD. We believe that this is the underlining justification for why DOP does not precisely predict the system impact of PMD when higher order PMD is present. Optical signals with orthogonal polarizations would have the same DOP, but their system penalties may be quite different. We also demonstrate a novel approach to estimate the maximum eye-closure penalty asymmetry with three orthogonal polarizations on the Poincaré sphere.

\section{EXPERIMENTAL SETUP}

Fig. 1 shows the experimental setup for our symmetry study of DOP and eye-closure penalty for orthogonal polarizations. An intensity-modulated $10-\mathrm{Gb} / \mathrm{s}$ pseudorandom binary sequence optical signal is generated by an Agilent tunable laser and a JDS-Uniphase Mach-Zehnder modulator. The optical signal passes through a PMD emulator (PMDE), which consists of either one stage or two stages of polarization-maintaining (PM) fiber to emulate first-order or higher order PMD. The statistical distribution of the PMD is not a consideration for this letter. After traversing the PMDE, the signal is amplified before entering a fiber link. The output signal from the fiber link is further amplified by a second erbium-doped fiber amplifier, optically filtered and fed into an Agilent high-speed oscilloscope equipped with a wideband optical detector. The optical filter 3-dB bandwidth is $1 \mathrm{~nm}$ and the optical signal-to-noise ratio of the optical signal is approximately $25 \mathrm{~dB}$ measured with a resolution bandwidth of $0.1 \mathrm{~nm}$. Eye-closure penalty has been used to characterize the PMD-induced system impairment 
[7] and is adopted in this letter. For any practical purpose, $Q$ parameter penalty can be considered to be proportional to eye-closure penalty. Subsequently, the measured relationship between eye-closure penalty and DOP can be considered to that between $Q$ penalty and DOP. The signal waveform traces are averaged to remove the random noise and recorded. Then the waveform data is converted into eye diagrams through a Matlab program to compute the eye-closure penalty due to PMD-induced waveform distortion. The signal Stokes vector is measured using an Agilent polarization analyzer by tapping the optical signal before it enters the fiber link. Because the fiber link has negligible PMD, the DOP symmetry is not altered by further passing through the fiber link. The rationale to monitor the Stokes vector before the fiber link is that in order to measure the symmetry/asymmetry of orthogonal polarization pairs as a function of the fiber chromatic dispersion, several different spools of transmission fibers are used for the fiber link during the measurement. By placing the polarization analyzer before the transmission fiber, the stability of the PMDE is monitored. The first polarization controller PC1 is used to optimize the input polarization for the Mach-Zehnder modulator, and the second polarization controller PC2 is used to systematically adjust the launch polarization into the PMDE for PMD/Stokes vector analysis. There is an additional PC before PC2 to ensure that the signal enters the half-wave plate (HWP) in PC2 with a linear polarization (not shown in Fig. 1). The systematic polarization adjustment is done by physically rotating the HWP in PC2 for $90^{\circ}$, which corresponds to the Stokes vector rotating of $360^{\circ}$ along a maximum circle on Poincaré sphere.

Both the DOP and the eye-closure penalty are functions of launch polarization (or input Stokes vector) into PMDE. Although the results can be presented by a three-dimensional polar plot, it would be quite complex and the intuitive picture might be lost. Instead, we show a two-dimensional polar plot presenting the DOP or eye-closure penalty as a function of the input polarization. When the launch polarization is rotated from $0^{\circ}$ to $360^{\circ}$ on the Poincaré sphere, the corresponding DOPs are measured with the polarization analyzer, and the signal waveform traces detected at the end of the transmission fiber spool are recorded, from which the eye-closure penalty is computed.

\section{MEASuREment Results And Discussion}

Fig. 2(a) and (b) shows the symmetry measurement for the eye-closure penalty with the transmission fiber chromatic dispersion of 0 and $-1360 \mathrm{ps} / \mathrm{nm}$ respectively. The negative dispersion of $-1360 \mathrm{ps} / \mathrm{nm}$ is obtained with a dispersion-compensation module (DCM-80) which is designed to compensate the dispersion of $80-\mathrm{km}$ standard single-mode fiber (SSMF). In this measurement, only one stage of PM fiber is used in the PMDE with 50-ps DGD and therefore only first-order PMD is involved. In Fig. 2(a), 0-km transmission fiber is used and each pair of orthogonal polarizations is represented by two data points which are $180^{\circ}$ apart on the two-dimensional polar plot. For instance, the measurements made at $80^{\circ}$ and $260^{\circ}$ is one of the orthogonal polarization pairs, represented by $A$ and $B$ in Fig. 2(a). We can see that all the orthogonal polarization pairs result in the same penalty, as evidenced by the symmetry of the data points for orthogonal pairs, for instance, data point $A$ is almost an inversion of $B$ across the origin. Such symmetry remains intact when $80 \mathrm{~km}$ of DCM fiber is introduced, as shown in Fig. 2(b). We also measure the symmetry of eye-closure penalty with 26,50 ,

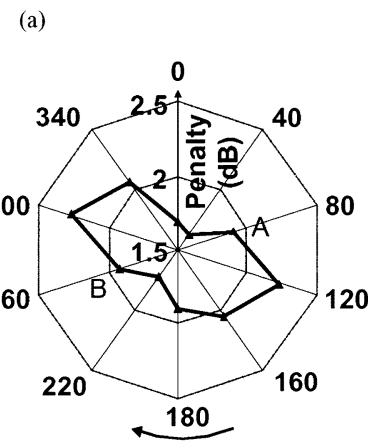

Rotation Angle ( $\left.{ }^{\circ}\right)$ (b)

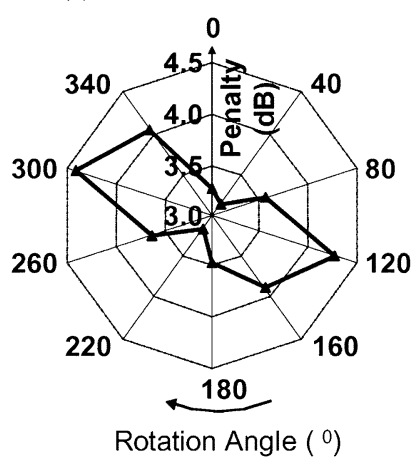

(c)

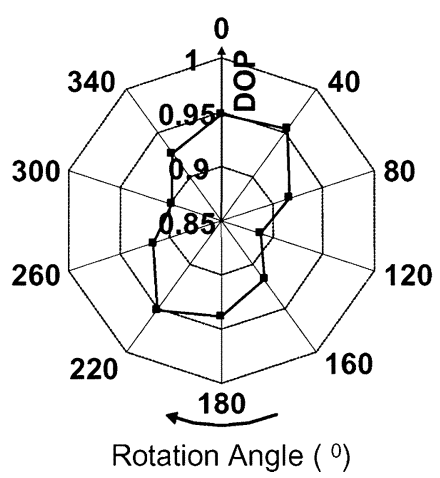

Fig. 2. Polar plots for (a) the eye-closure penalty for $0-\mathrm{km}$ fiber, (b) the eye-closure penalty for 80-km dispersion-compensation-module (DCM) fiber, and (c) the DOP for 0-km fiber. The tests are done with a single-stage 50-ps PM fiber. The dashed lines in the figure are the equal value contours for eye-closure penalty or DOP. Points A and B in Fig. 2(a) is one example of a pair of orthogonal polarizations. (a)

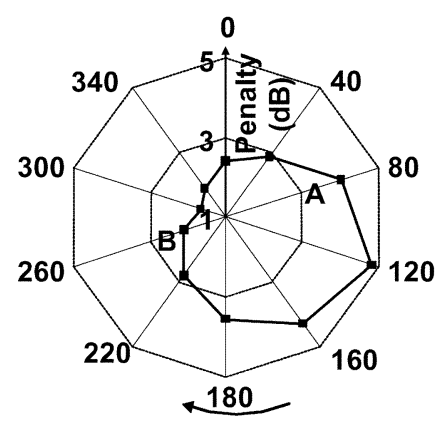

Rotation Angle ( ${ }^{\circ}$ ) (b)

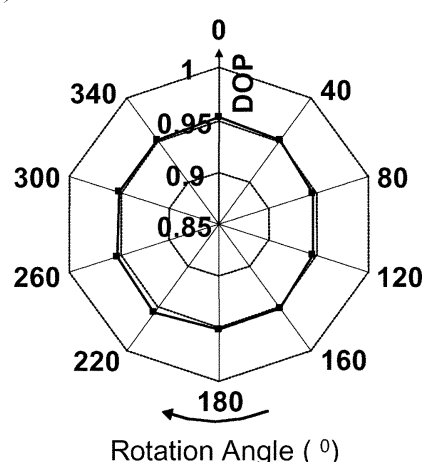

Fig. 3. Polar plot for (a) eye-closure penalty for $80-\mathrm{km}$ DCM fiber, and (b) DOP. The tests are done with two-stage PM fiber as a PMDE.

and 76-km SSMF, corresponding to the chromatic dispersion of 442,850 , and $1292 \mathrm{ps} / \mathrm{nm}$, respectively. The penalty symmetry remains intact for varying chromatic dispersion values we use. Similarly, Fig. 2(c) shows the measured DOP on the polar plot, and clearly each orthogonal polarization pair has the same DOP.

Fig. 3(a) shows the symmetry study for eye-closure penalty when the optical signal passes through a two-stage $(50+50 \mathrm{ps}$ DGD) PMDE with the eigen-axes of the two stages misaligned with each other so as to generate higher order PMD. Mathematically, the asymmetry is defined as the difference of the eye-closure penalty between a pair of orthogonal polarizations, which can be shown as [5]

$$
\begin{aligned}
\Delta P & =A \cdot\left|D_{e} \vec{J} \cdot \overrightarrow{\Omega^{\prime}}\right| \\
D_{e} & =D_{f}-\frac{2 \pi c}{\lambda^{2}} \phi_{0}^{\prime \prime}
\end{aligned}
$$


where $\Delta P$ is the eye-closure penalty asymmetry between an orthogonal polarization pair, $A$ is a coefficient dependent on various system parameters, $\vec{J}$ is input polarization to the PMD medium, $\overrightarrow{\Omega^{\prime}}$ is the second-order PMD, $\phi_{0}^{\prime \prime}$ is the second derivative of original phase of the pulse over the angular frequency [5], and $D_{e}$ is the effective chromatic dispersion including the contribution from fiber chromatic dispersion $D_{f}$ and original phase chirp $\phi_{0}^{\prime \prime}$. For a Gaussian pulse with an root-mean-square pulsewidth of $T_{0}$ and a chirp parameter of $\alpha, \phi_{0}^{\prime \prime}$ will be equal to $\left(2 \alpha T_{0}^{2}\right) /\left(1+\alpha^{2}\right)$. As we can see, the asymmetry of the eye-closure penalty is proportional to the magnitude of the effective chromatic dispersion and second-order PMD. Therefore, only the result obtained with large chromatic dispersion of $-1360 \mathrm{ps} / \mathrm{nm}$ from 80-km DCM is shown in Fig. 3(a). We see that even though the DOP is still symmetric for each orthogonal polarization pair as shown in Fig. 3(b), the symmetry of the eye-closure penalty is broken. For example, the penalties for the orthogonal pair at the angles of $120^{\circ}$ and $300^{\circ}$ are 1.7 and $4.8 \mathrm{~dB}$, respectively. This represents a $3-\mathrm{dB}$ asymmetry despite an identical DOP of $95 \%$ at these two launch polarizations. This further illustrates the inaccuracy of DOP as a PMD degradation monitor. The first-order PMD and second PMD are measured to be $33 \mathrm{ps}$ and $1500 \mathrm{ps}^{2}$, respectively, in this experiment.

We next quantitatively investigate the eye-closure penalty asymmetry for different fiber chromatic dispersions when the two-stage (50 + 50 ps DGD) PMDE are still used. From (1), the maximum asymmetry $\Delta P_{\max }$ occurs when the two launch polarizations are in- or out-of-phase with the second-order PMD vector, given by

$$
\Delta P_{\max }=A \cdot\left(D_{f}-\frac{2 \pi c}{\lambda^{2}} \phi_{0}^{\prime \prime}\right) \cdot\left|\overrightarrow{\Omega^{\prime}}\right| .
$$

In general, the maximum asymmetry may not be reached for orthogonal polarizations, but it can be measured by launched three orthogonal pairs, for instance, 1$)(1,0,0)$ and $(-1,0,0), 2)$ $(0,1,0)$ and $(0,-1,0)$, and 3$)(0,0,1)$ and $(0,0,-1)$ on the Poincaré sphere. It is easy to show that from (1) and (3)

$$
\Delta P_{\max }=\sqrt{\left(\Delta P_{1}\right)^{2}+\left(\Delta P_{2}\right)^{2}+\left(\Delta P_{3}\right)^{2}}
$$

where $\Delta P_{1-3}$ is the asymmetry of eye-closure penalty associated with one of the three orthogonal pairs on the Poincaré sphere. From (3), the maximum penalty asymmetry can be computed with the penalty values at these three pairs of orthogonal polarizations. Additionally, the maximum eye-closure penalty asymmetry is a linear function of the chromatic dispersion with the slope proportional to the second-order PMD [(3)]. In our experiment, we measure the eye-closure penalties for the three orthogonal pairs, and use (3) to derive maximum eye-closure penalty asymmetry. Fig. 4 shows the maximum penalty asymmetry at chromatic dispersions of 0,442 , and $850 \mathrm{ps} / \mathrm{nm}$. The solid line is the straight-line fit for the experimental data. Interestingly, the minimum penalty asymmetry occurs at $-195 \mathrm{ps} / \mathrm{nm}$ due to the chirp induced by the optical modulator, which is independently verified. We use the same coefficients to predict the eye-closure asymmetry for the chromatic dispersion

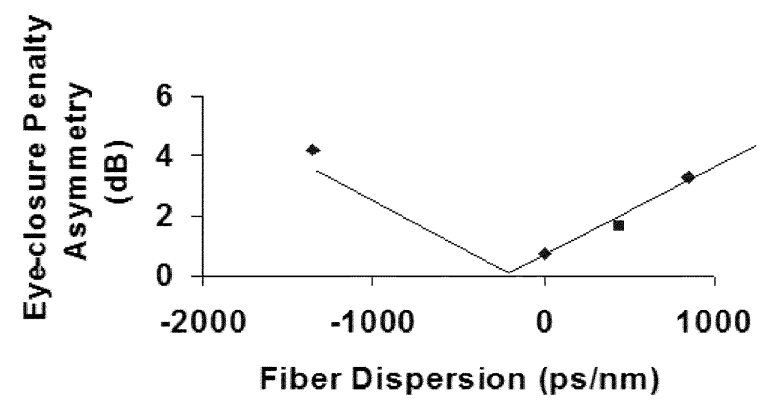

Fig. 4. Maximum eye-closure penalty asymmetry as a function of fiber dispersion.

of $-1360 \mathrm{ps} / \mathrm{nm}$ from $80 \mathrm{~km}$ of DCM fiber. The measured data shows $4.2-\mathrm{dB}$ asymmetry compared with the $3.5-\mathrm{dB}$ asymmetry predicted by theory. The difference is attributed to the relatively large eye-closure penalty around $6 \mathrm{~dB}$ for the $80-\mathrm{km}$ DCM fiber along with the impact of second-order PMD. The DOPs of three orthogonal pairs for each chromatic dispersion of transmission fiber are also recorded, showing intact symmetry in spite of corresponding large eye-closure asymmetry.

\section{CONCLUSION}

We perform a systematic measurement of the DOP and eyeclosure penalty for optical signals with orthogonal polarizations. We find that the symmetry of the DOP is intact under higher order PMD, whereas the symmetry of eye-closure penalty is broken under second-order PMD. An orthogonal polarization pair can have large disparity of eye-closure penalty despite an identical DOP. We also demonstrate a novel approach to estimate the maximum asymmetry of eye-closure penalty with three orthogonal polarizations on the Poincaré sphere.

\section{ACKNOWLEDGMENT}

The authors would like to thank K. E. Cornick at the University of Melbourne for her insightful discussion and valuable laboratory assistance.

\section{REFERENCES}

[1] C. D. Poole and R. E. Wagner, "Phenomenological approach to polarization dispersion in long single-mode fibers," Electron. Lett., vol. 22, pp. 1029-1030, 1986.

[2] C. Poole, R. W. Tkach, A. R. Chraplyvy, and D. A. Fishman, "Fading in lightwave systems due to polarization-mode dispersion," IEEE Photon. Technol. Lett., vol. 3, no. 1, pp. 68-70, Jan. 1991.

[3] N. Kikuchi, "Analysis of signal degree of polarization degradation used as control signal for optical polarization mode dispersion compensation,' J. Lightw. Technol., vol. 19, no. 4, pp. 480-486, Apr. 2001.

[4] W. Shieh, "Principal states of polarization for an optical pulse," IEEE Photon. Technol. Lett., vol. 11, no. 6, pp. 677-679, Jun. 1999.

[5] W. Shieh, "On the second-order approximation of PMD," IEEE Photon. Technol. Lett., vol. 12, no. 3, pp. 290-292, Mar. 2000.

[6] L. S. Yan, Q. Yu, T. Luo, A. E. Willner, and S. Yao, "Higher-order PMD compensation using a polarization controller and phase modulator in the transmitter," in OFC 2002, pp. 298-299.

[7] J. H. Winters and M. A. Santoro, "Experimental equalization of polarization dispersion," IEEE Photon. Technol. Lett., vol. 2, no. 8, pp. 591-593, Aug. 1990. 